\title{
A Medicina Interna do Centro Hospitalar Universitário S. João na Pandemia COVID-19
}

\author{
Internal Medicine in Centro Hospitalar Universitário S. João and the \\ COVID-19 Pandemic
}

\author{
Jorge Almeida (https://orcid.org/0000-0002-1337-7179)
}

Palavras-chave: Coronavírus; COVID-19; Infecções por Coronavírus; Medicina Interna; Pandemia

Keywords: Coronavirus; Coronavirus Infections; COVID-19; Internal Medicine; Pandemics

No início de dezembro de 2019, um novo coronavírus, denominado severe acute respiratory syndrome coronavirus-2 (SARS-CoV2), é descrito em Wuhan, China, resultando numa epidemia. A Organização Mundial de Saúde (OMS) denomina essa doença como coronavirus disease 2019 (COVID-19). Em 21 janeiro de 2020 é assumida a transmissão da doença entre humanos.

De acordo com a casuística de um centro chinês, divulgada em 7 de fevereiro, $41 \%$ dos doentes com COVID-19 tinham adquirido a infeção na instituição hospitalar (nosocomial) e, de forma alarmante, a doença tinha atingido $29 \%$ dos profissionais de saúde envolvidos. ${ }^{1}$ Sabíamos que a maior parte (mais de $80 \%$ ) dos doentes com infeção conhecida tinha doença ligeira, $14 \%$ tinham doença mais grave e 5\% apresentavam doença em estádio crítico. Aproximadamente 10\% dos doentes diagnosticados necessitariam de internamento hospitalar por pneumonia por SARS-CoV2, e desses, cerca de 10\% necessitariam de admissão em unidades de cuidados diferenciados (intermédios ou intensivos), alguns requerendo ventilação invasiva por síndrome de dificuldade respiratória aguda (ARDS)2 A mortalidade era mais comum nos indivíduos mais idosos e naqueles com comorbilidade (doenças cardiovasculares, diabetes mellitus, doença respiratória crónica, insuficiência renal e doenças oncológicas, entre outras), embora também pudesse atingir indivíduos mais jovens e sem comorbilidade, provocando doença crítica, disfunção múltipla de órgãos e eventualmente a morte.

A 5 de março o governo italiano toma medidas de confinamento, perante a rutura do sistema de saúde, face à COVID19; a divulgação dessas circunstâncias, pelos vários meios de

Diretor Serviço Medicina Interna, Centro Hospitalar Universitário S. João, Porto, Portugal Professor auxiliar convidado da FMUP

https://revista.spmi.pt - DOI: 10.24950/rspmi/ COVID19/J.Almeida / CHUSJOAOS/2020 comunicação e redes sociais, criou em todos os agentes de saúde um real e fundamentado alarmismo.

Dada a sua transmissibilidade, em semanas a doença atinge todos os continentes, exceto a Antártida, e é assumida como uma pandemia, pela Organização Mundial de Saúde (OMS), em 11 de março. ${ }^{3}$

\section{Plano de contingência do centro hospita- lar universitário S. João}

A 2 de março foi internado no Centro Hospitalar Universitário S. João (CHUSJ) o primeiro doente diagnosticado com COVID-19, coincidindo com outro caso internado na mesma data, em outro Hospital da cidade, tendo sido os dois primeiros casos descritos em território nacional.

Entretanto, no início de fevereiro, reconhecendo a gravidade da situação que vinha a ser descrita, o Conselho de Administração (CA) do CHUSJ, antecipou a necessidade de organização da resposta integrada da instituição, colmatando lacunas, antecipando aquisição de equipamentos e estabelecendo os vários planos de funcionamento em situação de contingência. Para tal, foi constituído um Gabinete de Crise, composto pela estrutura dirigente do Centro Hospitalar e dos serviços clínicos e não clínicos considerados prioritários na resposta imediata à COVID-19, nos quais se incluía, pela sua dimensão, capacidade e diferenciação, o Serviço de Medicina Interna (SMI). Esse Gabinete, em reuniões diárias, foi acertando e ajustando estratégias, de acordo com o conhecimento progressivo da realidade e das várias expressões da doença e seu potencial envolvimento. Nas primeiras semanas foram estabelecidas, revistas e divulgadas as várias normas de atuação e procedimentos, fundamentais na alteração dos comportamentos e na adaptação de todo o Centro Hospitalar, que estava apontado como pilar fundamental da região Norte e do País na resposta à pandemia, que se antecipava e se sentia poder ser devastadora. Ao mesmo tempo, foi sendo providenciada a dotação de recursos técnicos com aquisição de material de consumo clínico e aparelhos essenciais ao apetrechamento adequado dos serviços envolvidos.

Neste processo, foi absolutamente determinante o papel do CA que, de forma incansável, sempre assumiu a sua condução em sintonia com os clínicos e os técnicos envolvidos, que foram sempre consultados e cujas propostas iam sendo integradas nos sucessivos ajustamentos do plano estratégico. 
Era consensual o objetivo comum - prepararmo-nos o melhor possível, no mais curto espaço de tempo, para a resposta global que era de nós esperada, objetivo que, analisando a posteriori, conseguimos na plenitude. Acreditamos ter antecipado, nessa fase, em cerca de 3 semanas, a resposta dada pelo país, o que justifica a capacidade e recursos estratégicos que fomos revelando.

Assinalam-se algumas das centenas de determinações do CA que se tornaram particularmente relevantes, pela data ou pelo impacto futuro.

A aquisição de equipamentos de proteção individual (EPI) e de testes moleculares para identificação do SARS-CoV2, foi providenciada logo na primeira semana de fevereiro.

Em 12 de fevereiro foi estabelecido o primeiro procedimento para casos suspeitos detetados em qualquer local do CHUSJ. Ao mesmo tempo, foi iniciada a remodelação das áreas de atendimento do Serviço de Urgência (SU) e ajustados os equipamentos e materiais, de modo a criar um circuito independente para atendimento de doentes com suspeita de COVID-19. Mais tarde, foi criada uma estrutura específica para testes ("Tenda COVID-19") e aumentada a estrutura física do SU, com a montagem de uma área suplementar de $250 \mathrm{~m}^{2}$, concluída a 23 de março. Em 28 de março, foi instalado um aparelho de tomografia computorizada (TC) na área do SU, permitindo a realização de TC torácica a doentes com suspeita de COVID-19, de acordo com um protocolo elaborado por um conjunto de clínicos, em que também esteve envolvido o SMI.

$\mathrm{Na}$ primeira semana de março foi restringido o acesso geral ao CHUSJ e a atividade de ambulatório, com passagem da grande maioria das consultas à forma não presencial; ao mesmo tempo, foram estabelecidos os procedimentos do Serviço de Saúde Ocupacional para os profissionais com suspeita de infeção por SARS-CoV2, ou que contactaram com casos suspeitos ou confirmados de COVID-19, que foram sendo atualizados.

Na segunda semana de março, foi suspensa toda a atividade clínica não urgente, nomeadamente a atividade cirúrgica programada.

A 15 de março foi determinado o uso de máscara de proteção cirúrgica por todos os funcionários dentro da instituição e, em 6 de abril, também por todos os utentes, acompanhantes e visitantes autorizados em situações especiais.

Desde o início, foi assumida como essencial a disponibilidade, realização e tempo de resposta dos testes diagnósticos, com o Serviço de Patologia Clínica a priorizar os doentes internados e profissionais da instituição (orientados pelo Serviço de Saúde Ocupacional) - opção fundamental para minimizar a transmissão nosocomial e garantir a sustentabilidade da atividade hospitalar. A partir de 20 de março, têm sido realizados mais de 1200 testes diários, dos quais, a partir de meados de abril, cerca de 65\% são de resposta a necessidades externas à instituição - de outras estruturas do SNS, autarquias e instituições de solidariedade social.
O CHUSJ, desde a atribuição do estatuto de EPE, em 2005, implementou um modelo de gestão em que os serviços clínicos têm uma geometria variável, centrada na resposta às necessidades dos doentes e comunidade, à sua tipologia, rompendo a tradicional barreira territorial entre serviços, ou seja, quando deixa de haver vagas no espaço físico atribuído a cada serviço, os doentes são alocados noutros serviços que ainda as tenham. Assim se explica que o SMI, que dispõe de cerca de 200 camas de internamento em regime de enfermaria, tenha atingido no mês de janeiro, pontualmente, só nas enfermarias do polo Porto, a lotação de 285 camas. Para a gestão de admissões e alocação dos doentes, o CHUSJ conta com uma equipa específica de enfermeiros, que faz essa gestão nas 24 horas dos 365 dias do ano, desde há 4 anos.

Este modelo de gestão permitiu a execução dos vários planos delineados, e foi facilitado pela redução quase súbita da necessidade de internamento das restantes especialidades da área médica e pelo esvaziamento rápido de quase todas as enfermarias da área cirúrgica, muito dependentes da atividade programada.

Assim, foi determinada a alocação dos doentes com COVID-19, em regime de enfermaria, a vários serviços, sequencialmente, de acordo com as necessidades: inicialmente, parte do Serviço de Doenças Infeciosas e, em segundo tempo, todas as enfermarias (23 camas); seguiu-se o Serviço de Ginecologia; a 16 de março, foi a vez do SMI que, em duas fases, contribuiu com 2 setores, num total de 57 camas; de seguida, o Serviço de Cirurgia Vascular; depois, uma enfermaria de Cirurgia Geral e, por fim, uma enfermaria de Ortopedia (reservada a doentes em pós-operatório e com COVID-19). Nesta fase, tínhamos mais de 200 camas de enfermaria para doentes com infeção por SARS-CoV2 e tínhamos mais três enfermarias adaptadas para resposta em caso de necessidade (nos Serviços de Ortopedia, de Pneumologia e uma terceira do Serviço de Medicina Interna).

Entretanto, os Serviços de Otorrinolaringologia e Oftalmologia foram reservados para um internamento de curta duração de doentes do âmbito da Medicina Interna a aguardar o resultado de testes diagnósticos de COVID-19 (muitas vezes repetidos, quando o índice de suspeita se mantinha muito elevado, apesar da negatividade dos testes iniciais), determinante do local de alocação do doente - enfermaria de Medicina Interna ou enfermaria COVID-19.

Todos estes setores foram adaptados para o tratamento de doentes com COVID-19, com o apoio da Unidade de Prevenção e Controlo da Infeção e Resistência aos Antimicrobianos (UPCIRA), criando circuitos "limpos" e "áreas contaminadas" em cada uma das coortes, permitindo a circulação de profissionais com e sem equipamento de proteção individual de nível 2 (EPI-2).

Para a assistência clínica aos doentes com COVID-19, foi delineado, com o CA, um modelo de equipas mistas, constituídas por 4 elementos de várias especialidades, destacadas, em 
cada dia, para cada uma das várias coortes. Foram estabelecidas como especialidades centrais nessas equipas multidisciplinares, a Medicina Interna, a Infeciologia e a Pneumologia; a estas foram sendo associados especialistas e IFE de outras especialidades médicas e até cirúrgicas, bem como 3 IFG, de acordo com as necessidades em cada momento. A gestão logística destas equipas ficou a cargo do Serviço de Doenças Infeciosas, embora nas coortes do SMI fossem constituídas preferencialmente por elementos do próprio Serviço.

Desde a admissão dos primeiros doentes com COVID-19, foram definidos circuitos próprios para a sua circulação dentro do Hospital, para exames auxiliares de diagnóstico (privilegiando horários específicos para os não urgentes) ou terapêuticas específicas, desde a admissão e até ao momento da alta, com trajetos e elevadores específicos. O primeiro circuito para casos suspeitos foi estabelecido em 12 de fevereiro e foi sendo atualizado, em função da localização das coortes de doentes.

Em 23 de março, a norma 004/2020 da DGS alarga a assistência a doentes com COVID-19 aos restantes hospitais do SNS, não envolvidos na primeira fase, e também aos Cuidados de Saúde Primários, a partir das 00:00 horas de 26 de março.

\section{O serviço de medicina interna}

O SMI do CHUSJ tem uma importante função assistencial, prestando cuidados do foro da Medicina Interna a uma vasta população, sobretudo da região Norte do país. Presta cuidados altamente diferenciados em diversas áreas da especialidade, apoiando os hospitais de menor dimensão, que não apresentem capacidade para todos os cuidados específicos no âmbito da especialidade.

No contexto da resposta à pandemia, foi claro, desde o início do processo, que, pela sua diferenciação e capacidade para lidar com a doença, o SMI teria de responder ao novo desafio, mas sem deixar de manter a resposta às diferentes patologias que normalmente trata nas enfermarias. Assim, foram sendo implementados, revistos e alterados, às vezes semanalmente, os planos necessários para garantir estes objetivos, integrados no plano de contingência do CHUSJ.

\section{ENFERMARIAS DE DOENTES COM COVID-19}

Em relação aos doentes com COVID-19, o SMI integrou, desde o início, as equipas mistas, tendo contribuído com 19 clínicos - AH com menos de 50 anos (incluindo 5 recém-especialistas), e IFE dos $4^{\circ}$ e $5^{\circ}$ ano de internato. Nas coortes instaladas no SMI, as equipas são constituídas preferencialmente por clínicos do próprio Serviço.

A 16 de março, o SMI passou a ter uma coorte de doentes com COVID-19; um setor de 29 camas de enfermaria foi adaptado para tratamento dessa tipologia de doentes, sendo dotado de pressão tendencialmente negativa. Coincidindo com o pico da pandemia em Portugal, foi necessário adaptar, em apenas 2 dias, mais um setor do SMI, com 28 camas, que nas semanas anteriores também já tinha sido dotado de pressão tendencialmente negativa, e que iniciou atividade em 26 de março.

Este setor ficou com uma tipologia de doentes bem definida: 14 camas reservadas para doentes provenientes do Serviço de Medicina Intensiva, com manutenção da necessidade de oxigenioterapia por cânula nasal de alto fluxo (HFNC) ou ventilação não invasiva (CPAP ou BiPAP); as outras 14 camas destinam-se a doentes com COVID19 e necessidade de suporte ventilatório, para os quais, consensualmente, esse foi considerado o nível máximo de cuidados, determinado pela situação clínica prévia e pelo prognóstico inerente. Esta coorte de doentes com COVID19 foi dotada de equipamentos de ventilação e dispositivos de HFNC, em número considerado apropriado (10 aparelhos VNI Philips VC-60, 5 aparelhos de HFNC e outros 15 aparelhos de VNI adequados a esta patologia, bem como máscaras de Boussignac), assim como de um aparelho de avaliação de gasometria, garantindo a possibilidade de tratar doentes com necessidade de técnicas de oxigenação avançadas. A gestão inicial ficou a cargo da Unidade de Cuidados Intermédios de Medicina (UCIM), responsável pela coordenação das vagas com o Serviço de Medicina Intensiva e pelo apoio logístico e técnico, quando necessário; mais tarde, a responsabilidade passou para uma AHG de Medicina Interna, com experiência no tratamento de doentes críticos e no manuseio de VNI, que passou a integrar diariamente a equipa desse setor.

\section{ENFERMARIAS DE DOENTES SEM COVID-19}

No que respeita aos doentes sem COVID-19, mantivemos a disponibilidade de cerca de 140 camas de enfermaria, além de uma enfermaria do Serviço de Cirurgia Geral, que ficou logística e administrativamente integrada no SMI, até pela eventual necessidade de ser reconvertida em coorte para doentes com COVID-19. Foi alterada a estrutura e também a equipa assistencial envolvida no tratamento destes doentes.

A taxa de ocupação desta estrutura de 140 camas inicialmente rondava os $40 \%$, mas, com o evoluir da pandemia, tem aumentando paulatinamente, na proporção inversa ao contrário da das coortes de doentes com COVID-19.

Nesta fase, as restantes especialidades médicas do CHUSJ (além da Medicina Interna, Doenças Infeciosas e Pneumologia), bem como a quase totalidade das especialidades cirúrgicas, passaram a ter uma atividade apenas residual, garantindo o Serviço de Urgência específico e a atividade de ambulatório, predominantemente em regime de consultas não presenciais; a atividade cirúrgica eletiva ficou restrita quase só às doenças oncológicas e condicionada pela dificuldade de garantir vaga de unidade de cuidados intensivos no pós-operatório, se necessário.

Desde o início da pandemia, tem sido clara a extrema dificuldade em excluir a infeção por SARS-CoV2, muitas 
vezes com necessidade de recurso a múltiplos testes, incluindo pesquisa em secreções brônquicas, com intervalos mínimos de 24 horas. Esta dificuldade manteve-se apesar da existência da área em que os doentes aguardavam o resultado do teste inicial ou da sua repetição, quando julgado necessário, antes da admissão no SMI. A decisão do CA de, a partir de 26 de março, testar obrigatoriamente todos os doentes que carecessem de internamento, independentemente do motivo, criou mais alguma segurança aos profissionais, mas, como sabíamos, não resolveu o problema. A possibilidade recente de passarmos a realizar "testes diagnósticos moleculares rápidos" não melhorou significativamente essa perceção, uma vez que a sensibilidade é semelhante à dos testes mais morosos, já de si relativamente baixa.

O SMI adotou como estratégia, desde o início, a separação desses doentes - com testes negativos, mas com elevado índice de suspeita clínica, determinado pelo contexto epidemiológico e/ou clínica respiratória e/ou TC torácica sugestiva - em enfermarias específicas, em que os doentes são abordados como tendo COVID-19, até à minimização dessa possibilidade; os profissionais prestam assistência com EPI nível 2 e foram estabelecidos horários específicos para a realização de exames.

Com a evolução da pandemia, e sobretudo a partir de 15 de abril, os doentes sem COVID-19 começaram a ser claramente mais numerosos do que aqueles com este diagnóstico. Assim, atualmente (última semana de abril), em estratégia consertada com o CA, iniciamos o plano de realocação de doentes sem COVID-19 a uma das áreas do SMI que alocava uma coorte de doentes com COVID-19. Nessa enfermaria, dadas as condições físicas (possibilidade de isolamento e pressão tendencialmente negativa) e de recursos humanos (formação específica prévia da equipa de enfermagem e assistentes operacionais) ficarão os doentes que mantêm suspeita clínica elevada, apesar de exames negativos para COVID-19.

Por fim, mantivemos a equipa de Urgência Interna (Polo Porto e Polo Valongo), com a mesma dimensão que sempre tivemos, garantindo a assistência clínica a todos os doentes não COVID-19 do nosso Centro Hospitalar e funcionando como ponte com o SU Polivalente.

\section{UNIDADES DE CUIDADOS DIFERENCIADOS (UCIM E UAVC)}

As unidades de cuidados diferenciados do SMI [Unidade de AVC (UAVC) e UCIM] também foram adaptadas para a resposta às exigências dos doentes nesta pandemia.

Assim, a UCIM, com 11 camas, ficou exclusivamente vocacionada para doentes com infeção por SARS-CoV2, incluindo os doentes com acidente vascular cerebral agudo sujeitos a terapêutica de reperfusão ou com critérios para internamento em unidade de AVC, contando com a colaboração dos clínicos da UAVC na sua abordagem.
Esta unidade, já capacitada com os profissionais e recursos adequados ao tratamento da COVID-19 neste nível de cuidados, foi dotada com pressão negativa, garantindo o tratamento destes doentes em condições de segurança para os profissionais de saúde e, também aqui, foram criados circuitos "limpos" e "áreas contaminadas" permitindo a circulação dos referidos profissionais. A unidade passou a receber doentes provenientes das várias unidades de cuidados intensivos, em continuidade de cuidados, bem como doentes que, se viessem a necessitar de ventilação invasiva, seriam transferidos para uma das unidades de cuidados intensivos.

A abordagem destes doentes tem sido sempre adaptada, de acordo com as orientações que vão sendo disponibilizadas pelas várias sociedades científicas internacionais, usando como estratégias essenciais as técnicas de oxigenação (HFNC, CPAP e VNI, com recurso a equipamentos Philips VC-60 e Trilogy 202, e prone positioning) e a otimização da terapêutica médica.

Para além dos doentes com pneumonia por COVID-19, são admitidos nesta unidade todos os doentes com infeção por SARS-CoV2 e com necessidade de internamento em cuidados intermédios por qualquer outro motivo (síndromes coronárias agudas, taqui ou bradiarritmias, insuficiência cardíaca aguda, hemorragias digestivas, só para mencionar alguns).

A UAVC manteve a sua missão, assegurando a via verde do AVC do CHUSJ no período noturno e admitindo doentes com AVC agudo sem infeção por SARS-CoV2. Além disso, por ser uma unidade do SMI, com 5 especialistas de Medicina Interna no seu quadro clínico, também ficou com capacidade e disponibilidade para admitir doentes com necessidade de internamento em unidade de cuidados intermédios, se as restantes unidades do CHUSJ com essa tipologia não dispusessem de vagas; contaria com o apoio dos clínicos da UCIM sempre que necessário.

\section{O AMBULATÓRIO}

$\mathrm{Na}$ área de ambulatório, mantivemos a atividade na Consulta de Medicina Interna e nas outras oito consultas especializadas do SMI, privilegiando as consultas não presenciais, conforme plano do Serviço e determinação do CA, com a adesão quase total dos clínicos e dos doentes. No formato de consulta não presencial, têm sido realizadas algumas primeiras consultas, apesar das dificuldades óbvias na nossa especialidade; foram realizadas consultas presenciais sempre que, por decisão criteriosa do médico, foi entendido serem inadiáveis.

Atualmente está em processo de aprovação um plano de reestruturação do Centro de Ambulatório, a implementar a partir de maio, que prevê o aumento das consultas presenciais (particularmente as primeiras consultas), mas continua a privilegiar as não presenciais, sempre que possível. 


\section{RECURSOS HUMANOS}

Em qualquer plano de reestruturação é fundamental a capacidade de adaptação dos profissionais às funções que vão sendo criadas e alteradas, de acordo com as necessidades em cada momento. A capacidade técnica, aliada ao profissionalismo, espírito de missão, resiliência e determinação, têm constituído, como era esperado, a chave do sucesso deste plano.

Na primeira fase, três especialistas do SMI fizeram formação aos médicos e enfermeiros do Serviço sobre as medidas de proteção individual (etiqueta respiratória, higienização e colocação e retirada dos vários EPI) e circulação nos circuitos definidos; mais tarde, a formação foi alargada aos restantes profissionais (assistentes operacionais, técnicos e assistentes sociais) envolvidos na abordagem da doença.

Ao mesmo tempo, além da reestruturação do espaço físico do SMl, para receber as coortes de doentes com COVID-19, procedeu-se à reestruturação das equipas médicas que ficaram responsáveis pela assistência aos doentes sem COVID-19. Com a integração dos médicos mais novos nas equipas mistas de assistência às coortes de doentes com COVID-19, foram criadas 8 tiras para a assistência aos doentes sem infeção por SARS-CoV2 (cerca de 140 camas de enfermaria); essas tiras são constituídas maioritariamente pelos especialistas com mais de 55 anos, incluindo um AHG em funções no Parlamento Europeu, que foi integrado na estrutura assim que, voluntariamente, se apresentou no Hospital, por IFE, na esmagadora maioria dos 2 primeiros anos do internato (de Medicina Interna e outras especialidades médicas, em período de estágio formativo no SMI) e IFG.

A Urgência Interna (Polo Porto e Polo Valongo) de Medicina Interna ficou a ser assegurada pelos elementos que garantem a assistência às enfermarias do SMl e por $5 \mathrm{AH}$ que ficaram a assegurar as noites e restantes turnos que, entretanto, estivessem em falta. No mês de abril, vários IFE e alguns especialistas de especialidades médicas integraram, ou disponibilizaram-se para integrar, estas equipas em alguns turnos.

É nestes setores do SMI, onde a dificuldade em excluir a presença de infeção por SARS-CoV2 se sente diariamente, apesar da repetição de testes diagnósticos em muitos casos, que a intervenção da Direção do Serviço (DS) tem sido mais determinante, gerindo a exiguidade de EPI, na fase inicial, $e$ o misto de sentimentos, transversais a todas as classes profissionais, de, por um lado, ansiedade e receio de adquirir a doença e de contaminar os familiares, e por outro, o profissionalismo e espírito de missão, que efetivamente sempre prevaleceram. A adoção de medidas de isolamento de "suspeitos", a disponibilidade crescente de testes diagnósticos para COVID-19 e maior disponibilidade de outros exames subsidiários, a dotação de EPI em quantidade proporcional ao desejo da DS e a segurança clínica progressiva, com o conhecimento da doença, contribuíram para a estabilização rápida das incertezas criadas.
Apesar disso, e como era esperado, é neste setor que se têm verificado as ausências de profissionais, mais ou menos duradouras, tanto por doença COVID-19 como por necessidade de evicção laboral (doenças e risco inaceitável, períodos de isolamento profilático) com reflexo evidente na nossa atividade e capacidade de resposta. Assim, até à data, foram afetados 37 profissionais - 18 médicos, 15 enfermeiros e 4 assistentes operacionais; contraíram a doença, 25 profissionais - 9 médicos, 12 enfermeiros e os 4 assistentes operacionais. Não há qualquer registo de infeção por SARS-CoV2 de médicos do SMI integrados nas coortes de doentes com COVID-19.

O modelo assistencial com equipas multidisciplinares demonstrou ser vantajoso, por permitir uma capacidade de resposta proporcional, pelo ajustamento fácil e rápido às necessidades (variação do número de coortes de doentes com COVID-19), uma vez que, partindo de um ou dois elementos do núcleo de especialidades definidas como fundamentais, podem ser integrados outros clínicos com formação menos abrangente - por exemplo, vários especialistas da área cirúrgica integraram as equipas, desde o primeiro dia. Outro fator positivo do modelo, foi a partilha de conhecimento e a criação de espírito de grupo estre clínicos de várias especialidades, que, numa instituição com a dimensão da nossa, só esta pandemia promoveu.

Como aspetos negativos deste modelo, destaco a efetiva falta de continuidade de cuidados e a desmotivação que pode condicionar nos clínicos, pois equipas diferentes podem prestar assistência a diferentes coortes de doentes em dias sucessivos, com o tipo de cuidados a refletir também a diferenciação dos clínicos envolvidos. Por outro lado, o modelo de áreas "limpas" e "contaminadas" em cada coorte tem sido muito consumptivo de recursos humanos, tanto médicos, como de enfermagem e de assistentes operacionais, exigindo uma duplicação em cada tarefa - metade tem de estar na "área limpa" a apoiar os elementos que estão na "área contaminada"; entre os enfermeiros e assistentes operacionais, houve necessidade de realocar profissionais de outros serviços de especialidades médicas, cirúrgicas e do ambulatório, que tinham ficado com atividade assistencial reduzida.

Uma palavra de apreço pelo trabalho e disponibilidade dos médicos em período de formação, tanto de Medicina Interna como de outras especialidades médicas e cirúrgicas (no total, mais de 200 IFE e IFG), que constituíram um grupo designado Comissão de IFE, de cujo núcleo coordenador fazem parte 2 IFE de Medicina Interna. Desse modo se organizam para, além das atividades que muitos mantêm no contexto dos serviços a que pertencem (como os de Medicina Interna, Doenças Infeciosas e Pneumologia), colmatarem as várias necessidades no que respeita a escalas assistenciais ou outras solicitações. A relevância deste trabalho mereceu o reconhecimento do CA e o Grupo de IFE passou a integrar ativamente o Gabinete de Crise. 


\section{Abordagem da patologia}

A COVID-19, como nova entidade nosológica, tem constituído, naturalmente, um desafio.

Mesmo sentindo e tendo formação que os capacita para a abordagem dos doentes com COVID-19, os internistas do SMI têm procurado avidamente, a informação científica que vai surgindo, apesar de muitas vezes, pela velocidade e premência com que vai sendo disponibilizada, não ter a sustentação adequada. O SMI criou uma pasta partilhada na web, gerida por dois AH (uma delas em licença de maternidade) e o Diretor de Serviço, mas com a participação notória de todos, que compila e atualiza a informação científica.

Para a abordagem farmacológica, apesar da falta de evidência científica, mas respondendo à urgência de intervenção, e no seguimento da orientação da DGS, a Unidade de Farmacologia Clínica do CHUSJ emitiu uma orientação em que aconselhou a utilização de hidroxicloroquina ou, pontualmente, os inibidores da interleucina-6 (inibidores IL-6), em situações específicas. Requeremos a utilização de remdesivir num doente em fase inicial da doença e sem uso de ventilação invasiva, mas não foi disponibilizado; trata-se de um fármaco entendido como promissor, mas com um estudo recente desanimador, que pensamos ser menos eficaz se apenas for usado em fases avançadas da doença, como aconselhado pela DGS.

Entretanto, não há dúvida de que a oxigenação é fundamental: inicialmente por métodos convencionais e, se necessário, recorrendo a sistemas avançados de disponibilização de oxigenioterapia, de que o CHUSJ dispõe, desde o início da pandemia, também no SMI.

A capacidade de ventilação invasiva instalada no CHUSJ foi aumentada significativamente (numa $1^{\text {a }}$ fase, só a maior unidade de cuidados intensivos passou de 16 para 32 camas e as restantes - dos Serviços de Medicina Intensiva, Doenças Infeciosas e Anestesia - também foram reforçadas) e, felizmente, nunca foi necessário acionar o último nível do plano de contingência. Apesar disso, o recurso a ventilação invasiva foi sempre cuidadosamente avaliado, não só em função de necessidade imperiosa, mas também da evidência crescente de potencial efeito deletério.

A taxa de letalidade da COVID-19 no CHUSJ, até à data de 29 de abril, é de 2,6\%.

Esta é a visão, necessariamente breve, pelas características do artigo, do maior Serviço de Medicina Interna do nosso País, integrado na região do País mais atingida pela COVID-19, sobre a imperiosidade e necessidade de adaptação que esta pandemia condicionou, com algumas reflexões, que veiculam unicamente a nossa perspetiva, de algumas vertentes da nossa cada vez mais abrangente e diversificada atividade, como especialidade central e fundamental na dinâmica hospitalar.

\section{Responsabilidades Éticas}

Conflitos de Interesse: Os autores declaram a inexistência de conflitos de interesse na realização do presente trabalho.

Fontes de Financiamento: Não existiram fontes externas de financiamento para a realização deste artigo.

Proveniência e Revisão por Pares: Comissionado; sem revisão extema por pares.

\section{Ethical Disclosures}

Conflicts of interest: The authors have no conflicts of interest to declare. Financing Support: This work has not received any contribution, grant or scholarship.

Confidentiality of Data: The authors declare that they have followed the protocols of their work center on the publication of data from patients.

Provenance and Peer Review: Commissioned; without externally peer reviewed.

(c) Autor (es) (ou seu (s) empregador (es)) 2019. Reutilização permitida de acordo com CC BY-NC. Nenhuma reutilização comercial.

(c) Author(s) (or their employer(s)) 2019. Re-use permitted under CC BYNC. No commercial re-use.

\section{Correspondence/Correspondência: Jorge Almeida}

jorge.salmeida@hsjoao.min-saude.pt

Serviço de Medicina Interna, Centro Hospitalar Universitário S. João, Porto, Portugal Alameda Hernâni Monteiro, 4200-319 Porto

\section{Received/Recebido: 02/05/2020}

Accepted/Aceite: 02/05/2020

\section{Publicado / Published: 8 de Maio de 2020}

\section{REFERÊNCIAS}

1. Wang D, Hu B, Hu C, Zhu F, Liu X, Zhang J, et al. Clinical characteristics of 138 hospitalized patients with 2019 novel coronavirus-infected pneumonia in Wuhan, China. JAMA. 2020 (in press). doi: 10.1001/jama.2020.1585.

2. Wu Z, McGoogan JM. Characteristics of and important lessons from the Coronavirus disease 2019 (COVID-19) outbreak in China: Summary of Report of 72314 cases from the Chinese Center for Disease Control and Prevention. JAMA. 2020 (in press). doi: 10.1001/jama.2020.2648.

3. Organização Mundial de Saúde. Declaração OMS. [consultado 25 Abril 2020] Disponivel em: https://www.who.int/dg/speeches/detail/who-director-general-s-opening-remarks-at-the-media-briefing-on-covid-19---11-march-2020. 